\title{
Identification of Leishmania spp. promastigotes in the intestines, ovaries, and salivary glands of Rhipicephalus sanguineus actively infesting dogs
}

\author{
Milena Araúz Viol $^{1}$ - Felix D. Guerrero ${ }^{2}$ Bruno César Miranda de Oliveira ${ }^{1}$. \\ Monally Conceição Costa de Aquino ${ }^{1}$. Saulo Hudson Loiola ${ }^{1}$ • \\ Guilherme Dias de Melo ${ }^{1}$ - Aparecida Helena de Souza Gomes ${ }^{3}$. \\ Cristina Takami Kanamura ${ }^{4}$ Marcos Valério Garcia ${ }^{5}$. Renato Andreotti ${ }^{5}$. \\ Valéria Marçal Félix de Lima ${ }^{1}$ - Katia Denise Saraiva Bresciani ${ }^{1}$
}

Received: 24 March 2016 / Accepted: 3 May 2016/Published online: 12 May 2016

(C) Springer-Verlag Berlin Heidelberg (outside the USA) 2016

\begin{abstract}
Sand flies are recognized as the major vector of canine visceral leishmaniasis. However, in some areas of Brazil where sand flies do not occur, this disease is found in humans and dogs. There has been speculation that ticks might play a role in transmission of canine visceral leishmaniasis and the DNA of Leishmania spp. has been reported in whole ticks. We investigated the presence of Leishmania spp. promastigotes in the intestines, ovaries, and salivary glands of Rhipicephalus sanguineus ticks collected from tickinfested dogs in two cities of Brazil. We used 66 dogs that tested positive and 33 that tested negative for Leishmania spp. according to direct cytological examination assays. Ten ticks were collected from each dog and dissected to collect the
\end{abstract}

Electronic supplementary material The online version of this article (doi:10.1007/s00436-016-5111-5) contains supplementary material, which is available to authorized users.

Felix D. Guerrero

felix.guerrero@ars.usda.gov

1 Faculdade de Medicina Veterinária de Araçatuba, UNESP, Universidade Estadual Paulista Júlio de Mesquita Filho, Araçatuba, São Paulo, Brazil

2 United States Department of Agriculture, Agricultural Research Service, Knipling-Bushland U. S. Livestock Insects Research Laboratory, Kerrville, TX, USA

3 Centro de Laboratório Regional de Sorocaba, Instituto Adolfo Lutz, Sorocaba, São Paulo, Brazil

4 Centro de Patologia, Instituto Adolfo Lutz, São Paulo, São Paulo, Brazil

5 Empresa Brasileira de Pesquisa Agropecuária (EMBRAPA), Gado de Corte, Campo Grande, Mato Grosso do Sul, Brazil intestines, ovaries, and salivary glands for immunohistochemistry (IHC) and diagnostic real-time polymerase chain reaction (RT-PCR). IHC results showed Leishmania spp. in 98, 14, and $8 \%$ of the intestines, ovaries, and salivary glands, respectively. Real-time PCR showed that 89,41 , and $33 \%$ of the tick intestine, ovary, and salivary glands, respectively, were positive for Leishmania spp. The verification of promastigotes of Leishmania spp. by two independent techniques in ticks collected from these urban region dogs showed that there is need for clarification of the role of ticks in the transmission of canine visceral leishmaniasis in Brazil.

Keywords Ixodids · Leishmaniasis · Canine · Immunohistochemistry $\cdot$ Real-time PCR

\section{Introduction}

The dog is the main urban reservoir of visceral leishmaniasis, a zoonosis of considerable relevance to public health. The causal agent, Leishmania spp., is transmitted by the bite of sand flies (World Health Organization 2005), and human health problems of morbidity and mortality from leishmaniasis occur in 98 countries (World Health Organization 2014). Interestingly, Michalsky et al. (2009) reported that some areas in Brazil do not have sand flies, but visceral leishmaniasis occurs in both humans and dogs in these areas. Thus, other ectoparasites, such as fleas and ticks, have been suspected of playing major roles in transmission of leishmaniasis in the sand fly-free regions of Brazil.

The sand fly, Lutzomyia longipalpis, is the main vector of Leishmania infantum and visceral leishmaniasis in Brazil 
(Deane and Deane 1962). However, Leishmania spp. has been detected in fleas (Coutinho and Linardi 2007; Ferreira et al. 2009; Colombo et al. 2011) and ticks (Coutinho et al. 2005; Dantas-Torres et al. 2010a; Colombo et al. 2011; Trotta et al. 2012; Solano-Gallego et al. 2012; Morais et al. 2013) removed from dogs infected by leishmaniasis. Generally in these studies, PCR was used to indicate the presence of Leishmania spp. in the flea or tick samples. However, the role of these ectoparasites in the transmission or epidemiology of leishmaniasis cannot be definitively assigned solely by whole organism polymerase chain reaction (PCR). The gut of the ectoparasite would harbor remnants of blood directly ingested from feeding upon a leishmania-infected dog and this blood could serve as the direct reason for the PCR-positive result (Coutinho and Linardi 2007; Dantas-Torres et al. 2010a; Paz et al. 2010; Dantas-Torres 2011).

Rhipicephalus sanguineus, the brown dog tick, is a globally distributed ectoparasite which can ingest several types of pathogens while taking its blood meal, including Ehrlichia, Anaplasma, Babesia, Leishmania, and Hepatozoon (DantasTorres 2008). The role of this tick in the transmission of Leishmania has not been established despite several reports describing the detection of Leishmania DNA in $R$. sanguineus ticks removed from dogs naturally infected with $L$. infantum (Dantas-Torres et al. 2010a; Trotta et al. 2012; Solano-Gallego et al. 2012; Morais et al. 2013). Conversely, the study by Paz et al. (2010) concluded that it was unlikely that Leishmania maintenance and multiplication occurs within $R$. sanguineus. Our research group has focused upon the study of the epidemiology and diagnosis of canine visceral leishmaniasis, with an interest in the role nontraditional vectors might have in the biological cycle of this disease (Coelho and Bresciani 2013). We utilized immunohistochemistry (IHC) and PCR to test for the presence and prevalence of Leishmania spp. promastigotes in $R$. sanguineus ticks removed from tick-infested dogs in two urban areas of Brazil. Both sand flies and canine visceral leishmaniasis occur in Araçatuba, São Paulo State (SP), and Campo Grande, Mato Grosso do Sul State (MS), and those two cities were the source of infected dogs. Dogs were tested for their infection status regarding Leishmania spp. Engorged female ticks were removed from each dog and dissected, such that tick intestines, ovaries, and salivary glands could be individually tested by IHC and RT-PCR. Both the IHC and RTPCR assays found that a large percentage of tick tissues contained Leishmania spp. DNA and/or promastigotes.

\section{Material and methods}

\section{Approval by the ethics committee}

This study was approved by the Ethics Committee on Animal Use (CEUA) of the School of Agricultural and Veterinary
Sciences of UNESP, Universidade Estadual Paulista, Jaboticabal Campus, Process No. 011800/11.

\section{Canine selection and testing}

A total of 99 adult dogs, 65 male and 34 female, most of undefined breed and infested with ticks, were used in the study. The aim was to include canine visceral leishmaniasis-positive dogs from two endemic urban areas where each dog was infested by at least 10 ticks. We also included canine visceral leishmaniasis-negative dogs from one urban area not endemic for the disease. Cytological testing (described below) verified the presence or absence of the amastigote stage in the lymph nodes and/or bone marrow of each dog. The data from this testing can be found in Online Resource 1. Of 66 positive animals, 33 came from the Zoonosis Control Centers in the city of Campo Grande, MS, and 33 from the city of Araçatuba, SP, two areas endemic for canine visceral leishmaniasis. All 33 dogs negative for this infection were from the Center for Animal Health Research, CPPAR, UNESP, Jaboticabal, SP, which is generally considered a nonendemic area for canine visceral leishmaniasis (Sakamoto et al. 2007). The popliteal or pre-scapular lymph nodes were sampled using 5-ml syringes and $25 \times 7-\mathrm{mm}$ needles such that multiple aspirations were obtained from the same puncture. Bone marrow material was collected from the iliac crest, using 20-ml syringes and size $40 \times 12-\mathrm{mm}$ needles. Cytological prints on microscope slides were stained with Fast Panoptic (Laborclin, Pinhais, PR, Brazil) and the direct parasitological examinations for the Leishmania amastigote forms performed at $\times 100$ magnification (Troncarelli et al. 2009).

\section{Collections and dissections of ticks}

Ten engorged female $R$. sanguineus ticks were collected from each dog, and immediately rinsed in buffered saline solution pH 7.4 for $5 \mathrm{~min}$, in alcohol for $5 \mathrm{~min}$, then air-dried at room temperature on paper towels. All 10 ticks from each dog were pooled together for collection of dissected organ material. Ticks were dissected within $1-2 \mathrm{~h}$ after removal from the dog, mounting the ticks on Petri plates with paraffin. The intestines, ovaries, and salivary glands were removed from all 10 ticks and placed into microtubes containing buffered saline solution, one tube for each tissue type. Dissections were carefully performed to prevent cross contamination or rupture of the organs and any ruptured organs were discarded (Edwards et al. 2009; Dantas-Torres et al. 2010a). After dissection, approximately half of the material in each tube was transferred to another microtube containing $1.5 \mathrm{ml}$ of $10 \%$ buffered formalin, $\mathrm{pH} 7.4$ for IHC. 


\section{IHC to detect LPG}

Tick ovaries, salivary glands, and intestines were fixed in formaldehyde, embedded in paraffin, and subjected to microtomy for the preparation of 3- $\mu \mathrm{m}$ thickness histological sections on silanized slides. The sections were deparaffinized, rehydrated, subjected to antigen recovery under humid heat, and immersed in $10 \mathrm{mM}$ citric acid solution $\mathrm{pH} 6.0$ in a pressure cooker for $3 \mathrm{~min}$ similar to the method described in Norton et al. (1994). After cooling, slides were rinsed in $\mathrm{H}_{2} \mathrm{O}$ and subjected to endogenous peroxidase blockage by incubation in 20 volumes of $6 \%$ hydrogen peroxide solution, at $37{ }^{\circ} \mathrm{C}$, for $30 \mathrm{~min}$. Final washes in $\mathrm{H}_{2} \mathrm{O}$ and phosphatebuffered saline solution (PBS) preceded storage at room temperature prior to IHC.

The sectioned tissues were incubated with mouse monoclonal primary anti-Leishmania lipophosphoglycan (LPG) antibody (clone CA7AE raised against Leishmania donovani promastigotes, Thermo Fisher Pierce Biotechnology, Rockford, IL, USA) diluted 1:10,000 in $1 \%$ bovine serum albumin in PBS at $4{ }^{\circ} \mathrm{C}$, for $18 \mathrm{~h}$ in a humid chamber without shaking. Sections were washed in PBS for 3-5 min at room temperature and the antigen-antibody complex amplified by the addition of a third generation polymer (Shi et al. 1999) tagged with anti-mouse and anti-rabbit immunoglobulins and peroxidase enzyme (Reveal Polyvalent HRP, Spring Biosciences, Pleasanton, CA, USA), using an incubation at $37^{\circ} \mathrm{C}$ for $30 \mathrm{~min}$ in a humid chamber according to the manufacturer's protocol. Excess unbound polymer was removed by washing for $3-5$ min at $37{ }^{\circ} \mathrm{C}$ with PBS. Reaction products were visualized by addition of chromogenic substrate $0.1 \%$ diaminobenzidine solution (Sigma-Aldrich, St. Louis, MO, USA) in $0.1 \%$ hydrogen peroxide in PBS and incubating for 3-5 min at $37^{\circ} \mathrm{C}$. Positive signals were evidenced by a golden brown color. Subsequently, slides were extensively washed in $\mathrm{H}_{2} \mathrm{O}$ and subjected to light counterstaining with Harris hematoxylin followed by mounting with cover slip and Entellan permanent medium (Merck, Darmstadt, Germany) for microscopy. Negative controls were obtained by omission of the primary antibody.

\section{DNA extraction from tick organs}

DNA samples were extracted by protocols similar as reported by Sangioni et al. (2005) and stored at $-80{ }^{\circ} \mathrm{C}$. Briefly, the organs were suspended in $150 \mu \mathrm{L}$ of buffer $(10 \mathrm{mM}$ Tris- $\mathrm{HCl}$, 1 mM EDTA, pH 8.0) and homogenized with $450 \mu \mathrm{L}$ of $5 \mathrm{M}$ guanidine thiocyanate. Following extraction with phenol and chloroform, the aqueous phase was recovered and nucleic acids precipitated with $3 \mathrm{M}$ sodium acetate and isopropanol at $-80{ }^{\circ} \mathrm{C}$ for $18 \mathrm{~h}$. The nucleic acids were recovered by centrifugation, rinsed in $70 \%$ ethanol, and the washed pellet dried at $56{ }^{\circ} \mathrm{C}$ in a thermoblock for $15 \mathrm{~min}$ with the tube cap opened. The pellet was resuspended in $50 \mu \mathrm{l}$ of $\mathrm{H}_{2} \mathrm{O}$, assisted by mixing and heating at $65^{\circ} \mathrm{C}$ for $15 \mathrm{~min}$ in a thermoblock. The DNA was quantified and stored at $-80^{\circ} \mathrm{C}$.

\section{Diagnostic RT-PCR}

The primers ITS1-F (5'-CCTATTTTACACCAACCCCCAGT$\left.3^{\prime}\right)$ and ITS1-R (5'-GGGTAGGGGCGTTCTGCGAAA-3') were used with protocols similar as reported in Perosso et al. (2014). The specificity of the primer was tested using the Primer-Blast program of the National Center for Biotechnology Information (Bethesda, MD, USA; http:// www.ncbi.nlm.nih.gov/tools/primer-blast/). These primers amplify the intergenic internal transcribed spacer region of the rRNA gene. RT-PCR was carried out in a Bio-Rad thermocycler (Bio-Rad, Hercules, CA, USA) in a total volume of $20 \mu \mathrm{l}$, which consisted of $1.0 \mu \mathrm{L}$ of each primer ( $10 \mathrm{pmol}), 1$. $0 \mu \mathrm{l}$ sample DNA, $7.0 \mu \mathrm{L} \mathrm{H} \mathrm{H}_{2} \mathrm{O}$, and $10 \mu \mathrm{L}$ of the Quantifast Syber Green mix (Qiagen, Valencia, CA, USA). Samples were run in duplicate on a program consisting of 2-min incubation at $50{ }^{\circ} \mathrm{C}$ followed by $95^{\circ} \mathrm{C}$ for $2 \mathrm{~min}$ and then 40 amplification cycles that each consisted of $95^{\circ} \mathrm{C}$ for $15 \mathrm{~s}$ followed by $60^{\circ} \mathrm{C}$ for $30 \mathrm{~s}$. The melt-curve protocol was $95^{\circ} \mathrm{C}$ for $15 \mathrm{~s}, 60^{\circ} \mathrm{C}$ for $15 \mathrm{~s}$, followed by a 20-min gradient step from 60 to $95{ }^{\circ} \mathrm{C}$ which was maintained for $15 \mathrm{~s}$.

\section{Statistical analysis}

For statistical analysis, McNemar's test (Zar 2010) was adopted to compare the proportion of positive results for Leishmania among the tick organ samples.

\section{Results and discussion}

Prior to designing and conducting the diagnostic RT-PCR assay, analysis of the prospective PCR primers by the PrimerBlast program verified that significant sequence similarity did not exist between the primers and DNA from Trypanosoma cruzi, Babesia spp., and Ehrlichia spp. All tick tissue pools obtained from each of the 33 dogs testing negative for Leishmania spp. by cytological testing also tested negative in both the RT-PCR and IHC assays of the dissected tissues (Online Resource 1).

The tick tissue pools of intestines, ovaries, and salivary glands obtained from the 66 dogs that tested positive for Leishmania spp. by cytological tests showed varying results in the IHC and RT-PCR assays (Online Resource 1). In this group consisting of all infected canines, positive IHC immunolabeling for Leishmania spp. was detected in 98.5, 13.6 , and $7.6 \%$ of the 66 pools of intestines, ovaries, and salivary glands, respectively. Figure 1 shows representative photographs of Leishmania spp. promastigotes in dissected 
Fig. 1 Photomicrographs representing positive immunolabeling of Leishmania spp. promastigotes in organs of $R$. sanguineus-engorged females. a Intestine. b Ovary. c Salivary gland (magnification $\times 100$ )
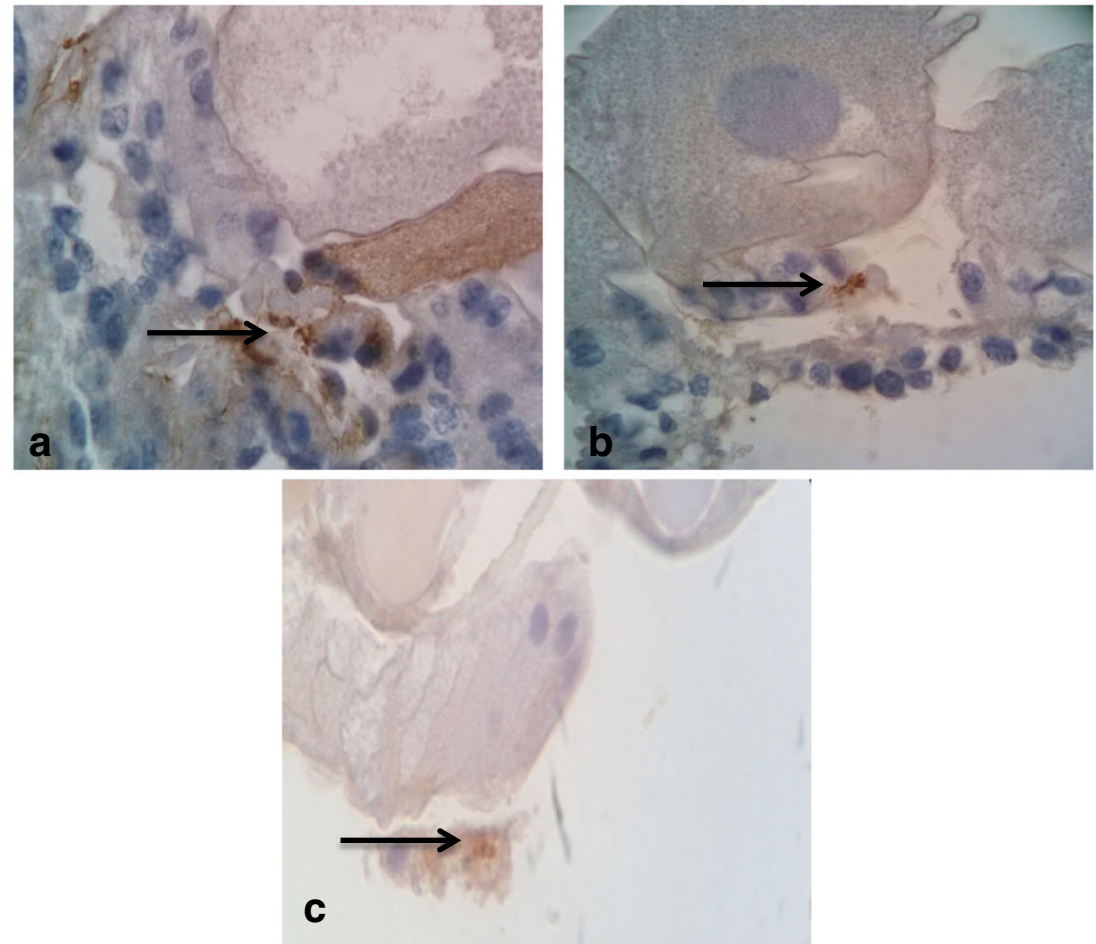

tick tissues. Diagnostic RT-PCR assay results from these pooled tick tissue samples showed that Leishmania spp. DNA was detected in $89.4,40.9$, and $33.3 \%$ of the intestines, ovaries, and salivary glands, respectively. Figure 2 graphically compares overall results of the IHC and RT-PCR tissue assays. The RT-PCR gave an approximately fourfold higher percentage of positives than the IHC for both ovary and salivary gland (Fig. 2). In the intestine samples, the IHC detected a slightly higher incidence of positives than the RT-PCR ( 98.5 vs. $89.4 \%$ ). This might be due to carryover of RT-PCR inhibitory substances that occur in blood-containing samples, such as the intestinal materials of our samples. A caveat in our using RT-PCR as a diagnostic assay with dissected tick materials is that cross-contaminating intestinal materials might have affected the ovary and salivary gland sample results. Engorged female ticks contain large amounts of ingested blood and extended intestinal organ that can be damaged and burst during dissections. Although care was taken to prevent tissue damage during dissections, damaged leaking intestinal material could have been the cause of the higher RTPCR-positive percentages in the ovary and salivary gland samples compared to the IHC.

Table 1 shows the IHC and RT-PCR results according to location. Interestingly, the ovary and salivary gland tissue pools from Campo Grande, MS, showed a greater level of positive results compared to the tissue pools from
Fig. 2 Comparison of results for the IHC (gray) and RT-PCR (black) assays of tick intestine, ovary, and salivary gland

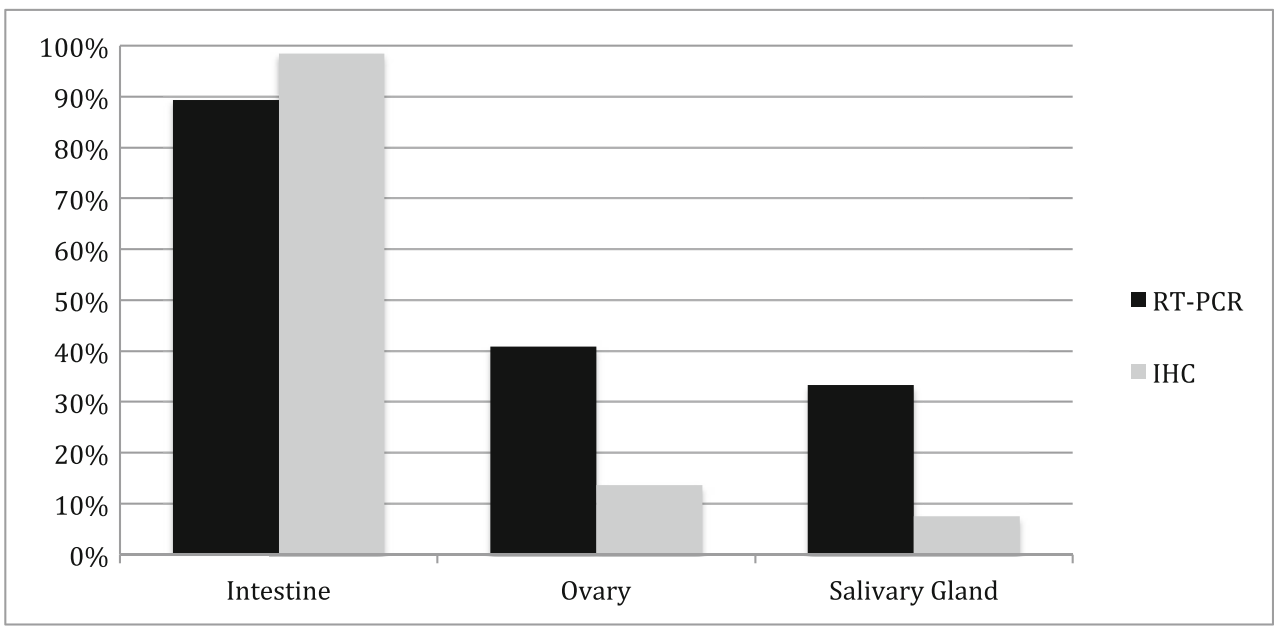


Table 1 IHC and RT-PCR assay results according to canine city of origin

\begin{tabular}{|c|c|c|c|c|c|c|c|}
\hline \multirow[t]{2}{*}{ City } & \multirow[t]{2}{*}{ No. of dogs } & \multicolumn{3}{|l|}{ RT-PCR } & \multicolumn{3}{|l|}{$\mathrm{IHC}$} \\
\hline & & Intestine & Ovary & Salivary gland & Intestine & Ovary & Salivary gland \\
\hline Campo Grande & 33 & 87.9 & 51.5 & 48.5 & 100 & 24.2 & 15.2 \\
\hline Araçatuba & 33 & 90.9 & 30.3 & 18.2 & 97.0 & 3.0 & 0 \\
\hline Jaboticabal & 33 & 0 & 0 & 0 & 0 & 0 & 0 \\
\hline
\end{tabular}

Araçatuba, SP. This occurred despite both cities showing similar results for the intestine samples. The reason for the higher positive results in the Campo Grande ovary and salivary gland samples is not known. Perhaps an environmental or biological factor differentially influences the developmental process of the Leishmania spp. within the canines of the two cities.

Our IHC technique used a primary LPG monoclonal antibody raised against $L$. donovani promastigotes as the molecule to interact with putative Leishmania spp. promastigotes in our samples. We selected this diagnostic assay because LPG is known to be present on the surface of the promastigote glycocalyx (Naderer et al. 2004; McConville and Blackwell 1991; Moody et al. 1993; Assis et al. 2012; Forestier et al. 2015). The promastigote form of all members of the genus Leishmania is believed to synthesize LPG, and its main glycoconjugate covers the entire parasite surface, including the flagellum (Turco and Descoteaux 1992). There is less known about LPG synthesis in the amastigote stage, although McConville and Blackwell (1991) reported that the amastigotes of $L$. donovani do not appear to synthesize LPG. This uncertainty in the synthesis of LPG by amastigotes limits our findings somewhat. However, Forestier et al. (2015) discuss the observation that the progressive development of promastigotes into amastigotes within a mammalian host is accompanied by a large downregulation of LPG expression. The specificity of our LPG antibody must be investigated further to allow us to unequivocally state that our IHC findings in the tick tissues relate specifically to the promastigote stage. The ability to distinguish between amastigotes and promastigotes is important because promastigotes develop within an arthropod host, whereas amastigotes develop within a mammalian host. Ticks feeding upon infected canines would ingest amastigotes present in the host blood. Assuming that the antibody is specific for the promastigote stage, our detection of promastigotes in tick ovaries and salivary glands provides evidence that $R$. sanguineus ticks can propagate Leishmania infections as alternate arthropod hosts feeding upon infected canines.

Detection of the Leishmania DNA by diagnostic PCR has been previously described in other Brazil-based studies. Although these studies tested smaller numbers of dogs and ticks compared to our study, Coutinho et al. (2005), Colombo et al. (2011), Morais et al. (2013), and Campos and Costa (2014) detected Leishmania DNA in 33, 50, 44, and $23 \%$ of sampled ticks, respectively. None of these studies used dissected ticks nor did they use an independent technique such as IHC to verify the presence of promastigotes in their Leishmania spp. RT-PCR-positive samples. Thus, these studies could not assess if the positive results were due to remnants of ingested Leishmania DNA in the intestines of the sampled ticks or if the positive results truly represented development of infective promastigotes within the tick. Our study is the first report using biologically relevant tick organ samples dissected from individual ticks to assay for Leishmania spp. with both molecular and immunohistological tools. It should be noted that a study of Ixodid ticks sampled from serologically positive dogs from Italy detected Leishmania in some tick salivary gland samples (Dantas-Torres et al. 2010a). Also, transovarian dissemination of Leishmania was detected by PCR assays performed on eggs from experimentally infected female ticks (Dantas-Torres et al. 2010b).

We expected to detect Leishmania spp. on ticks feeding upon infected dogs. The sensitivity of RT-PCR meant extra care was needed in the tick collection and template preparation process. Also, the feeding ticks are taking up infected blood into their intestines. We desired to detect if ticks could play biologically relevant roles in Leishmania transmission in regions where the sand fly vector is absent. By dissecting tick tissues, we found that a significant percentage of tick salivary glands had Leishmania spp. DNA and promastigotes (Figs. 1 and 2). Tick susceptibility to infection by Leishmania chagasi was proven by using inoculation via peritoneal and oral route in hamsters (Coutinho et al. 2005). This finding has particular importance when we consider that canines can eventually ingest ectoparasites, but this question still needs to be elucidated. To extend our knowledge and confirm the role of $R$. sanguineus as a vector of canine visceral leishmaniasis, it remains necessary to establish the tick's ability to support the growth, multiplication, and transstadial transmission of this protozoon. This could be accomplished using in vivo transmission studies in uninfected canines, using ticks harboring Leishmania promastigotes.

Acknowledgments We thank Fundação de Amparo à Pesquisa do Estado de São Paulo - FAPESP (processes: 2011/51113-4 and 2012/00266-8), for the financial support to this study. FDG acknowledges funding support from the US Department of Agriculture-Agricultural Research Service CRIS Project No. 3094-32000-036-00. USDA is an equal opportunity employer. 


\section{Compliance with ethical standards}

Conflict of interest The authors declare that they have no conflict of interests.

Ethical standards All experiments were conducted in a manner that complies with the current laws and regulations of Brazil.

\section{References}

Assis RR, Ibrain IC, Nogueira PM, Soares RP, Turco SJ (2012) Glycoconjugates in New World species of Leishmania: polymorphisms in lipophosphoglycan and glycoinositolphospholipids and interaction with hosts. Biochim Biophys Acta 1820:1354-1365

Campos JHF, Costa FAL (2014) Participation of ticks in the infectious cycle of canine visceral leishmaniasis, in Teresina, Piauí, Brazil. Rev Inst Med Trop 56:297-300

Coelho WMD, Bresciani KDS (2013) Molecular and parasitological detection of Leishmania spp. in a dipteran of the species Tabanus importunus. Rev Bras Parasitol Vet 22:605-607

Colombo FA, Odorizzi RMFN, Laurenti MD, Galati EAB, Canavez F, Pereira-Chioccola VL (2011) Detection of Leishmania (Leishmania) infantum RNA in fleas and ticks collected from naturally infected dogs. Parasitol Res 109:267-274

Coutinho MTZ, Linardi PM (2007) Can fleas from dogs infected with canine visceral leishmaniasis transfer the infection to other mammals? Vet Parasitol 147:320-325

Coutinho MT, Bueno LL, Sterzik A, Fujiwara RT, Botelho JR, De Maria M, Genaro O, Linardi PM (2005) Participation of Rhipicephalus sanguineus (Acari: Ixodidae) in the epidemiology of canine visceral leishmaniasis. Vet Parasitol 128:149-155

Dantas-Torres F (2008) The brown dog tick, Rhipicephalus sanguineus (Latreille, 1806); from taxonomy to control. Vet Parasitol 152:173-185

Dantas-Torres F (2011) Ticks as vectors of Leishmania parasites. Trends Parasitol 27:155-159

Dantas-Torres F, Lorusso V, Testini G, De Paiva-Cavalcanti M, Figueredo LA, Stanneck D, Mencke N, Brandão-Filho SP, Alves LC, Otranto D (2010a) Detection of Leishmania infantum in Rhipicephalus sanguineus ticks from Brazil and Italy. Parasitol Res 106:857-860

Dantas-Torres F, Martins TF, De Paiva-Cavalcanti M, Figueredo LA, Lima SB, Brandão-Filho SP (2010b) Transovarial passage of Leishmania infantum kDNA in artificially infected Rhipicephalus sanguineus. Exp Parasitol 125:184-185

Deane LM, Deane MP (1962) Visceral Leishmaniasis in Brazil: geographical distribution and transmission. Rev Inst Med Trop 4:198212

Edwards KT, Goddard J, Varela-Stokes AS (2009) Examination of the internal morphology of the Ixodid tick, Amblyomma maculatum Koch, (Acari: Ixodidae); a "how-to" pictorial dissection guide. Midsouth Entomol 2:28-39

Ferreira MGPA, Fattori KR, Souza F, Lima VMF (2009) Potential role for dog fleas in the cycle of Leishmania spp. Vet Parasitol 165:150-154

Forestier CL, Gao Q, Boons GJ (2015) Leishmania lipophosphoglycan: how to establish structure-activity relationships for this highly complex and multifunctional glycoconjugate? Front Cell Infect Microbiol 4:1-7

McConville MJ, Blackwell JM (1991) Developmental changes in the glycosylated phosphatidylinositols of Leishmania donovani. J Biol Chem 266:15170-15179
Michalsky EM, França-Silva JC, Barata RA, Lara E, Silva FO, Loureiro AM, Fortes-Dias CL, Dias ES (2009) Phlebotominae distribution in Janaúba, an area of transmission for visceral leishmaniasis in Brazil. Mem Inst Oswaldo Cruz 104:56-61

Moody SF, Handman E, McConville MJ, Bacic A (1993) The structure of Leishmania major amastigote lypophosphoglycan. J Biol Chem 268:18457-18466

Morais RCS, Gonçalves SC, Costa PL, Silva KG, Silva FJ, Silva RP, Brito MEF, Brandão-Filho SP, Dantas-Torres F, Paiva-Cavalcanti M (2013) Detection of Leishmania infantum in animals and their ectoparasites by conventional PCR and real time PCR. Exp Appl Acarol 59:473-481

Naderer T, Vince JE, McConville MJ (2004) Surface determinants of Leishmania parasites and their role in infectivity in the mammalian host. Curr Mol Med 4:649-665

Norton AJ, Jordan S, Yeomans P (1994) Brief, high-temperature heat denaturation (pressure cooking): a simple and effective method of antigen retrieval for routinely processed tissues. J Pathol 173:371379

Paz GF, Ribeiro MFB, Michalsky EM, Lima ACVMR, França-Silva JC, Barata RA, Fortes-Dias CL, Dias ES (2010) Evaluation of the vectorial capacity of Rhipicephalus sanguineus (Acari: Ixodidae) in the transmission of canine visceral leishmaniasis. Parasitol Res 106: 523-528

Perosso J, Silva KLO, Ferreira SIS, Avanço SV, Santos PSP, Eugênio FR, Almeida BFM, Lima VMF (2014) Alteration of sFAS and sFAS ligand expression during canine visceral leishmaniosis. Vet Parasitol 205:417-423

Sakamoto CAM, Bresciani KDS, Serrano ACM, Lima VMF, Machado GF, Kanamura CT, Costa AJ (2007) Canine Visceral Leishmaniasis at Jaboticabal—São Paulo State, Brazil-first case report. Ars Vet 23:125-128

Sangioni LA, Horta MC, Vianna MCB, Gennari SM, Soares RM, Márcio AM, Galvão MAM, Schumaker TTS, Ferreira F, Vidotto O, Labruna MB (2005) Rickettsial infection in animals and Brazilian spotted fever endemicity. Emerg Infect Dis 11:265-270

Shi SR, Guo J, Cote RJ, Young LL, Hawes D, Shi Y, Thu S, Taylor CR (1999) Sensitivity and detection efficiency of a novel two-step detection system (PowerVision) for immunohistochemistry. Appl Immunohistochem Mol Morphol 7:201-208

Solano-Gallego L, Rossi L, Scroccaro AM, Montarsi F, Caldin M, Furlanello T, Trotta M (2012) Detection of Leishmania infantum DNA mainly in Rhipicephalus sanguineus male ticks removed from dogs living in endemic areas of canine leishmaniosis. Parasit Vectors 5:98-102

Troncarelli MZ, Camargo JB, Machado JG, Lucheis SB, Langoni H (2009) Leishmania spp. and/or Trypanosoma cruzi diagnosis in dogs from endemic and nonendemic areas for canine visceral leishmaniasis. Vet Parasitol 164:118-123

Trotta M, Nicetto M, Fogliazza A, Montarsi F, Caldin M, Furlanello T, Solano-Gallego L (2012) Detection of Leishmania infantum, Babesia canis, and rickettsiae in ticks removed from dogs living in Italy. Ticks Tick Borne Dis 3:293-296

Turco SJ, Descoteaux A (1992) The lipophosphoglycan of Leishmania parasites. Annu Rev Microbiol 46:65-94

World Health Organization (2005) Division of control tropical disease. Leishmaniasis control. Geographical distribution. WHO/CTD. http://www.who.int/ctd/html/leisgeo.html. Accessed 30 April 2015

World Health Organization (2014) Leishmaniasis. http://www.who.int/ leishmaniasis/epidemic/706 response more/en/index.html. Accessed 30 April 2015

Zar JH (2010) Biostatistical analysis, 5th edn. Prentice-Hall, Upper Saddle River 